\title{
Laser-induced ultrafast insulator-metal transition in $\mathrm{BaBiO}_{3}$
}

\author{
Alexander E. Lukyanov, ${ }^{1,2}$ Vyacheslav D. Neverov, ${ }^{1,2}$ Yaroslav V. Zhumagulov, ${ }^{3,2,1}$ Alexey P. Menushenkov $\odot,{ }^{1}$ \\ Andrey V. Krasavin $\odot,{ }^{1}$ and Alexei Vagov ${ }^{2}$ \\ ${ }^{1}$ National Research Nuclear University MEPhI, Kashirskoye shosse 31, Moscow, 115409, Russian Federation \\ ${ }^{2}$ ITMO University, St. Petersburg 197101, Russian Federation \\ ${ }^{3}$ University of Regensburg, Regensburg, 93040, Germany
}

(Received 23 June 2020; revised 7 October 2020; accepted 26 October 2020; published 9 November 2020)

\begin{abstract}
We investigate ultrafast coherent quantum dynamics of undoped $\mathrm{BaBiO}_{3}$ driven by a strong laser pulse. Our calculations demonstrate that in a wide range of radiation frequencies and intensities the system undergoes a transient change from the insulating to the metallic state, where the charge density wave and the corresponding energy spectrum gap vanish. The transition takes place on the ultrafast timescale of tens femtoseconds, comparable to the period of the corresponding lattice vibrations. The dynamics are determined by a complex interplay of the particle-hole excitation over the gap and of the tunneling through it, giving rise to the highly nontrivial time evolution which comprises high harmonics and reveals periodic reappearance of the gap. The time evolution is obtained by solving the dynamical mean-field theory equations with the realistic parameters for the system and radiation. Results are summarized in the phase diagram, helpful for a possible experimental setup to achieve a dynamical control over the conduction state of this and other materials with the similarly strong electron-phonon interaction.
\end{abstract}

DOI: 10.1103/PhysRevResearch.2.043207

\section{INTRODUCTION}

Perovskite compounds based on $\mathrm{BaBiO}_{3}(\mathrm{BBO})$ are a special class of the high-temperature superconductors (HTSC) that do not include transition metal ions. As a rule, such structures have a complex phase diagram with many structural phases that depend on the doping level as well as on the temperature. These include dielectric, metallic and superconductive phases, with the critical temperature of the superconductive transition varying between $T_{c}=13 \mathrm{~K}$ for the led doped materials [1] and $T_{c}=34 \mathrm{~K}$ for the potassium doping [2].

Unique properties of those materials are related to their peculiar crystal structure and its dynamical distortions (phonons), and to the Fermi surface configurations. For example, when doped with potassium with the concentration $x \geqslant 0.37$, the local electronic structure is characterized by a spatially separated mixture of free electrons and localized electron pairs [3-5]. The electron pairs determine the charge transfer and formation of the superconductive state, while free electron states play a role in the insulator-metal phase transition and the appearance of the Fermi-liquid state above the percolation threshold [6].

Bismuthates are characterized by strong deformations of the crystal lattice associated with the anomalously high amplitude of their phonon oscillations [7]. In this context the

Published by the American Physical Society under the terms of the Creative Commons Attribution 4.0 International license. Further distribution of this work must maintain attribution to the author(s) and the published article's title, journal citation, and DOI. most interesting is the "breathing" phonon mode with alternating sizes of the $\mathrm{BiO}_{6}$ octahedra and $\mathrm{Bi}-\mathrm{O}$ bonding along three crystallographic directions [8,9] (see Fig. 1). The size alternation involves the charge transfer by electron pairs tunneling between the two neighboring octahedra. The octahedron with the extra electron pair is larger, whereas the one without it is smaller. This migration of electron pairs can be interpreted as the "valence disproportionation" where $\mathrm{Bi}^{3+}$ and $\mathrm{Bi}^{5+}$ ions are formed instead of two neighboring $\mathrm{Bi}^{4+}$ ions $[10,11]$. Different electronic filling of the Bi6s-O2 $p_{\sigma^{*}}$ orbitals of the neighboring $\mathrm{BiO}_{6}$ octahedra creates a dynamical double-well potential for the vibration of oxygen ions, experimentally observed in EXAFS spectra $[6,12]$.

The inherently strong coupling between electrons in the conduction band and lattice distortions profoundly affects the carriers state in those materials. Specifically, in the parent compound, $\mathrm{BaBiO}_{3}$ with the Fermi level in the middle of the conduction band this coupling gives rise to the charge density wave (CDW) accompanied by the optical gap in the single-particle electronic spectra of the system, estimated in various studies as being $\simeq 2 \mathrm{eV}[7,13-16]$.

Details of the electronic spectra as well as of the carrierphonon coupling in those materials can be probed by studying their properties when the system is externally driven from its equilibrium, creating the system dynamical response. For example, the gap structure of the spectrum is investigated by the pump-and-probe measurements of the optical conductivity (see Refs. $[17,18]$ ), where the charge carriers are excited above the gap leading to a transient metallic state, gradually relaxing towards the original insulating state by the electronhole recombination. The system can be driven so strongly that its original phase is transiently changed. 
(a)

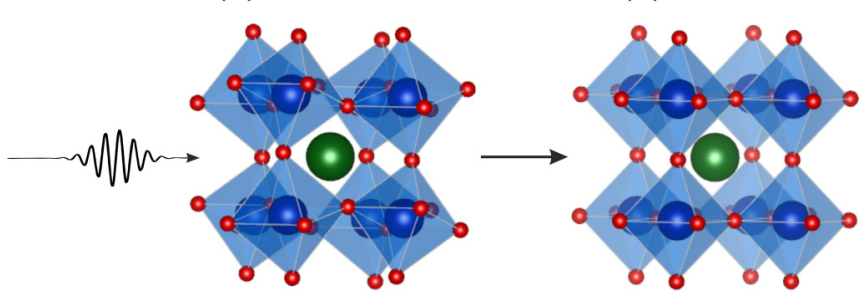

FIG. 1. A schematic crystal structure of $\mathrm{BaBiO}_{3}$, with ions $\mathrm{Bi}$ (blue), $\mathrm{O}$ (red), and $\mathrm{Ba}$ (green). (a) The structure before the pulse, distorted by the $\mathrm{CDW}$, has larger and smaller $\mathrm{BiO}_{6}$ octahedra, extra electron/hole pairs sit on the upper antibonding Bi6s-O2 $\mathrm{p}_{\sigma^{*}}$ orbitals. (b) The laser excitation breaks the pairs on the antibonding orbitals and removes the lattice distortion.

An adequate interpretation of such experiments requires modeling the dynamics far from the equilibrium. This has to be done beyond the single-particle picture since the system state is altered considerably. In addition, many relevant nonequilibrium phenomena take place on the ultrafast timescale, where quantum coherences play a large role and cannot be neglected.

Sufficiently accurate and consistent analysis of the ultrafast dynamic in strongly correlated systems, performed on equal footing with the analysis of their equilibrium state, became possible relatively lately, owing to rapid advances in the computing power. The dynamics of strongly correlated systems far from equilibrium have been addressed in a number of recent works, that studied, in particular, electron-mediated relaxation after ultrafast pumping [19], dynamical redistribution of spectra [20], the role of the phonon-carrier coupling in the time-resolved optical spectra [21], dynamics of the photodoped charge transfer insulators [22], the gap dynamics in excitonic insulators [23] and the dynamics of a periodically driven strongly correlated Fermi-Hubbard model [24].

The BBO is a representative example of the systems where the insulating CDW state appears as the result of the strong phonon-carrier coupling. Whether the $\mathrm{CDW}$ in this material can be externally manipulated and the metallic state achieved transiently by applying a strong driving is a very interesting problem, which until now has been investigated neither experimentally nor theoretically. Generally, little is known about the dynamics of this structure when it is driven far from equilibrium.

In this work, we investigate the ultrafast dynamics of the $\mathrm{BaBiO}_{3}$ excited by a strong continuous laser pulse. In our analysis we consider a time evolution of the system retaining all pertinent quantum coherences within the dynamical mean-field approach adapted to study strongly correlated systems $[25,26]$. The chosen level of the theory is sufficient to describe both the metal-insulator transition of the system and its ultrafast time evolution on the equal footing, ensuring consistency of the results.

Our analysis demonstrates that a sufficiently strong excitation can break the insulating state and the system evolves into the metallic phase on the timescale of tens femtoseconds. It is shown that the dynamics of this regime is highly nonlinear, comprises many high harmonics and even develops the contin- uous high-frequency spectra. Although our study focuses on $\mathrm{BaBiO}_{3}$, the results are general and can be used as a guidance for experimental setups to control the state of the system with the strong electron-phonon interaction.

The paper is organized as follows. In Sec. II, we introduce the model, describe the used assumptions, present necessary details of the formalism and discuss the choice of the system parameters. Results of the calculations are found in Sec. III in which we give examples of the various types of the dynamical behavior of the system as the reaction to the applied excitation field, and give a summary of the obtained results in the form of the phase diagram. Finally, Sec. IV briefly summarizes the results of the work and outlines future perspectives.

\section{MODEL AND METHODS}

\section{A. Holstein model for $\mathrm{BaBiO}_{3}$}

To describe the metal-insulator transition in $\mathrm{BaBiO}_{3}$, we adopt the tight-biding (TB) model for electrons that occupy $\mathrm{BiO}_{6}$ octahedra and can tunnel between them. The octahedra are regarded as "sites" and the hopping elements describe the tunneling. The coupling to the lattice deformation modes is taken into account within the Holstein model [27] where an electron of an octahedron is coupled to the distortion oscillation (phonon) mode of this octahedron. This model neglects the interaction between phonon modes of different octahedra. The Hamiltonian of the Holstein model is

$$
\begin{aligned}
H= & -\sum_{i j \sigma}\left(t_{i j} c_{j \sigma}^{\dagger} c_{i \sigma}+\text { H.c. }\right)-\mu \sum_{i}\left(n_{i \uparrow}+n_{i \downarrow}\right) \\
& +g \sum_{i}\left(b_{i}^{\dagger}+b_{i}\right)\left(n_{i \uparrow}+n_{i \downarrow}-1\right)+\omega_{\mathrm{ph}} \sum_{i} b_{i}^{\dagger} b_{i},
\end{aligned}
$$

where $c_{i \sigma}$ denotes the electron operators with spin $\sigma$ located at site $i, n_{i \sigma}=c_{i \sigma}^{\dagger} c_{i \sigma}$ is the electron number operator, $\mu$ is the chemical potential, $t_{i j}$ are the hopping integrals, $b_{i}$ is a phonon operator corresponding to the deformation mode on site $i, \omega_{\mathrm{ph}}$ is the mode frequency and $g$ is the phonon-electron coupling constant. Values of $t_{i j}$ are extracted from the ab initio calculations by comparing obtained single-particle density of states (DOS) with the results for the tight-biding model (1). The comparison yields that the tight-binding model needs to account for the nearest as well as the second- and the fourth-nearest neighbors. The corresponding hopping matrix elements are extracted as $t_{1}=-0.45 \mathrm{eV}, t_{2}=-0.09 \mathrm{eV}$, $t_{4}=0.10 \mathrm{eV}$, respectively, while the chemical potential is $\mu=-0.13 \mathrm{eV}$.

\section{B. Bethe lattice model}

The Holstein model (1) appears simple, however, is still quite difficult to solve, even within the dynamical mean-field theory (DMFT). However, one can simplify the calculations considerably by taking into account the details of the metalinsulator transition. The transition is associated with the CDW state where the size of the neighboring octahedra $\mathrm{BiO}_{6}$ alternate. The CDW state of interest is associated with the doubling of the unit cell of the simple cubic lattice of $\mathrm{BaBiO}_{3}$ in all three directions so that the corresponding $\mathrm{CDW}$ vector 


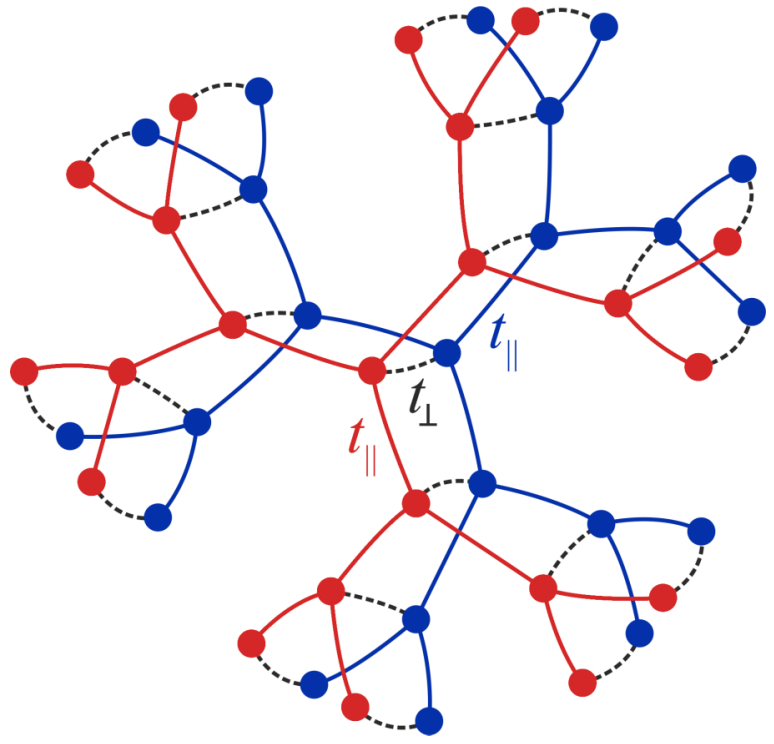

FIG. 2. Double Bethe lattice. Solid and dashed lines correspond to hoppings $t_{\|}$within and $t_{\perp}$ between the lattices, respectively. Each lattice is shown with coordination number 3 for clarity.

is $(\pi, \pi, \pi)$. This choice extracted from experimental observations for $\mathrm{BaBiO}_{3}[6,28,29]$.

The alternation of the neighboring sites and the appearance of the CDW state can be conveniently described by separating the original system into two sublattices of the double-size elementary cell, each containing octahedra of the same state. Within this model, the metal-insulator transition takes place when electronic states of the sublattices become different. The dynamics within sublattices is described using the Bethe lattice model, schematically illustrated in Fig. 2 for the coordinate number 3 . It is effectively infinite-dimensional model with a continuum of electronic states which gives rise to the quantum relaxation kinetics. The main advantage of the Bethe model is that its DMFT description yields exact results. It is commonly assumed that the Bethe lattice model offers a good approximation for 3D systems [25] and can be used as a trusted approximation to investigate dynamical properties of many-body systems (see, e.g., Refs. [26,30-33], and also Ref. [34] and references therein). Here it is adopted to describe the dynamics of each sublattice. At the same time the model with two Bethe sublattices is adequate to describe a CDW state with the broken symmetry between sublattices.

For the $\mathrm{BaBiO}_{3}$ structure of interest our final model consists of two equivalent Bethe lattices (see Fig. 2) where the electron can hope between the nearest neighbors of each sublattice (intralattice hopping) as well as between the equivalent sites of different sublattices (interlattice hopping). In the following, we refer to this model as the double Bethe lattice model (DBLM). The Hamiltonian of the model reads as

$$
\begin{aligned}
H= & -t_{\|} \sum_{\langle i, j\rangle \alpha \sigma} c_{j \alpha \sigma}^{\dagger} c_{i \alpha \sigma}-t_{\perp} \sum_{i \sigma}\left(c_{i 0 \sigma}^{\dagger} c_{i 1 \sigma}+\text { H.c. }\right) \\
& -\mu \sum_{i \alpha \sigma} n_{i \alpha \sigma}+g \sum_{i \alpha}\left(b_{i \alpha}^{\dagger}+b_{i \alpha}\right)\left(n_{i \alpha \uparrow}+n_{i \alpha \downarrow}-1\right) \\
& +\omega_{\mathrm{ph}} \sum_{i \alpha} b_{i \alpha}^{\dagger} b_{i \alpha}
\end{aligned}
$$

(a)

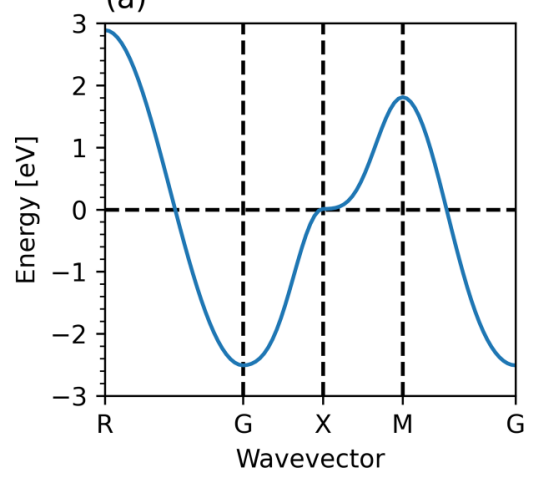

(b)

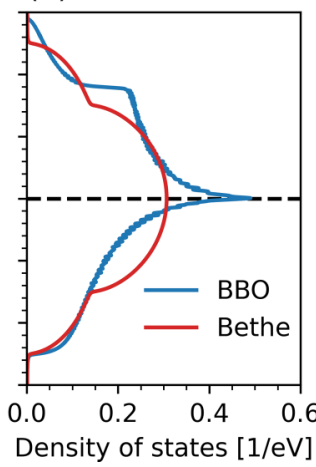

FIG. 3. (a) Band structure of the tight-binding model (1) for the cubic BBO structure [27]. (b) Single particle DOS calculated for the original TB model with the cubic BBO structure (blue) and for the double Bethe lattice model (red).

where $\alpha=0,1$ is the sublattice index, the sum with $\langle i, j\rangle$ goes over the nearest neighbor sites, $t_{\|}$and $t_{\perp}$ denote the intra- and interlattice hopping matrix elements, respectively. Their values are chosen such that the model yields the best possible approximation for the DOS. Figure 3 shows the band structure [panel (a)] and the DOS [panel (b)] of the TB model as well as the DOS of the DBLM. The best fit of the DOS is achieved for $t_{\|}=1 \mathrm{eV}$ and $t_{\perp}=0.5 \mathrm{eV}$. The Bethe lattice gives a qualitatively similar DOS but it does not capture the van Hove singularity in the middle of the band. The van Hove singularity facilitates the instability towards the formation of the CDW state when the Fermi level is close to it, $\mu \simeq 0$. However, the DBLM demonstrates this instability without the singularity in the DOS.

Notice that the dynamics of electrons with different spins as determined by the Hamiltonian (2) is independent and can be obtained by considering the spinless version of the model (2), where the carrier density $n_{i \alpha}$ enters the phonon coupling as $n_{i \alpha \uparrow}+n_{i \alpha \downarrow}=2 n_{i \alpha}$.

Finally, the coupling between the laser field and the system carriers is taken into account via the Peierls substitution, where the hopping integrals are assigned with the phase as $t_{i j} \rightarrow t_{i j} \exp \left(i \varphi_{i j}\right)$, calculated as the integral of the vector potential $\varphi_{i j}=(e / c \hbar) \int_{i}^{j}(\mathbf{A} \cdot d \mathbf{r})$ between the sites $i$ and $j$. In the calculations we assume that the external driving affects only the coupling between the sublattices with the corresponding hopping matrix elements

$$
t_{\perp}(t)=t_{\perp} e^{i \varphi(t)}, \quad \varphi(t)=f(t) \sin \left(\omega_{0} t\right),
$$

where $\omega_{0}$ is the frequency of the driving oscillations and $f(t)$ is the envelope function of the pulse. The approximation where the driving affects only the coupling between sublattices follows from the goal of our study that is to describe the nonlinear dynamics and the relaxation of the CDW state when the system is subject to laser pulses of high intensity. In the chosen model the laser excites dynamical degrees of freedom associated with the CDW. We note, however, that in the regime of strongly nonlinear dynamics of interest in this work all dynamical degrees of freedom (excitation modes), including those describing the dynamics within sublattices, are intermixed. Therefore, all such modes will be eventually 


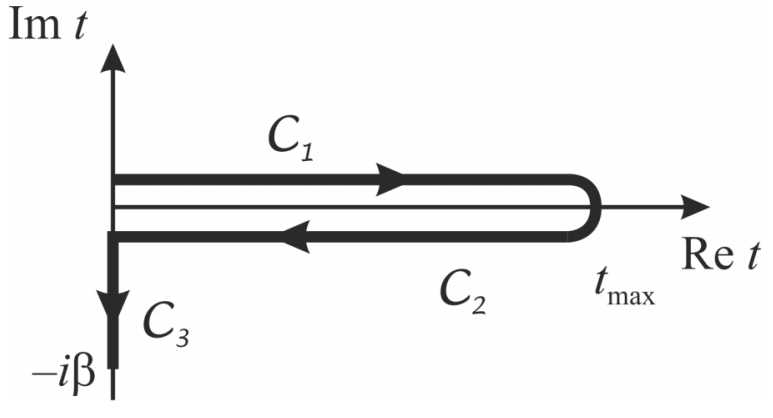

FIG. 4. Kadanoff-Baym contour with three time domains: realtime forward $\mathcal{C}_{1}$ and backward $\mathcal{C}_{2}$, and imaginary-time $\mathcal{C}_{3}$. The arrows set the contour time ordering; $t_{\max }$ corresponds to the maximum realtime propagation of the solution.

excited regardless of the driving type. Finally, in this work, we investigate the case of the constant driving so that the pulse arrives at time $t=0$ and then has a constant amplitude, $f(t)=\Theta(t) A_{0}$, where $A_{0}$ is the dimensionless driving strength and $\Theta(t)=1$ at $t>0$ and zero otherwise.

\section{DMFT for the Bethe lattice model}

To calculate the system dynamics we employ the DMFT approach [25], which maps the original many-body problem to a model with an effective impurity. Its application to the model with a single Bethe lattice has a number of crucial simplifications with respect to the original TB model [31]. Here we extend this approach to the DBLM in Eq. (2), where the impurity becomes a dimer, defined on both sublattices. The main DMFT equations in this case retain their general appearance, however, acquire a form of the $2 \times 2$ matrix equations for the corresponding matrix Green functions. For the convenience of the reader we briefly outline the main equations for the dynamics together with the used approximations.

All pertinent physical quantities of the system are found from the two-time electron $G\left(t, t^{\prime}\right)$ and phonon $D\left(t, t^{\prime}\right)$ matrix Green functions for the dimer impurity, which are obtained by solving the Dyson equations

$$
\begin{aligned}
& D\left(t, t^{\prime}\right)=D_{0}\left(t, t^{\prime}\right)+\int_{\mathcal{C}} d \tau d \tau^{\prime} D_{0}(t, \tau) \Pi\left(\tau, \tau^{\prime}\right) D\left(\tau^{\prime}, t^{\prime}\right), \\
& {\left[i \partial_{t}-\epsilon(t)\right] G\left(t, t^{\prime}\right)-\int_{\mathcal{C}} d \tau \Sigma(\tau, t) G(t, \tau)=\delta_{\mathcal{C}}\left(t, t^{\prime}\right),}
\end{aligned}
$$

where the integration is performed along the Kadanoff-Baym $(\mathrm{KB})$ contour $\mathcal{C}$ in Fig. 4 and $\delta_{\mathcal{C}}\left(t, t^{\prime}\right)$ is the $2 \times 2$ matrix delta function defined on this time contour. The phonon bare Green function is defined by

$$
D_{0}^{-1}\left(t, t^{\prime}\right)=-\frac{1}{2 \omega_{\mathrm{ph}}}\left(\partial_{t}^{2}+\omega_{\mathrm{ph}}^{2}\right) \delta_{\mathcal{C}}\left(t, t^{\prime}\right),
$$

and $\epsilon(t)$ is the one-particle Hamiltonian,

$$
\epsilon(t)=\left(\begin{array}{cc}
\mu+\Sigma_{0}^{M F}(t) & t_{\perp}(t) \\
t_{\perp}^{*}(t) & \mu+\Sigma_{1}^{M F}(t)
\end{array}\right)
$$

with the mean-field contribution to the self-energy

$$
\Sigma_{\alpha}^{\mathrm{MF}}(t)=g \bar{X}_{\alpha}(t),
$$

where the dimensionless quantity $\bar{X}_{\alpha}(t)=X_{\alpha}(t) \sqrt{2 M \omega_{\mathrm{ph}} / \hbar}$ ( $M$ is the oxygen mass) related to the sublattice displacement $X_{\alpha}(t)$, which measures the structural distortion of the octahedra and is calculated by averaging the phonon operators. The impurity self-energy for electrons in Eq. (4) comprises two components,

$$
\Sigma\left(t, t^{\prime}\right)=\Sigma_{\mathrm{ME}}\left(t, t^{\prime}\right)+\Delta_{G}\left(t, t^{\prime}\right),
$$

where the hybridization function $\Delta_{G}\left(t, t^{\prime}\right)$ in the case of the Bethe lattice has the form [25]

$$
\Delta_{G}\left(t, t^{\prime}\right)=t_{\|}^{2} G\left(t, t^{\prime}\right),
$$

whereas for the contribution $\Sigma_{\mathrm{ME}}$ to the self-energy beyond the mean-field we use the self-consistent Migdal approximation $[35,36]$

$$
\Sigma_{\mathrm{ME}}\left(t, t^{\prime}\right)=i g^{2} D\left(t, t^{\prime}\right) G\left(t, t^{\prime}\right) .
$$

For the phonon impurity self-energy this approximation yields

$$
\Pi\left(t, t^{\prime}\right) \rightarrow \Pi_{\mathrm{ME}}\left(t, t^{\prime}\right)=-2 i g^{2} G\left(t, t^{\prime}\right) G\left(t^{\prime}, t\right) .
$$

Solutions to the Dyson equations are used to obtain the average lattice displacement needed for the self-consistent solution of the Dyson equations

$$
\begin{aligned}
\bar{X}_{\alpha}(t)= & -\frac{2 g}{\omega_{\mathrm{ph}}}\left[2 n_{\alpha}(0)-1\right] \\
& +2 g \int_{0}^{t} d \tau D_{0, \alpha \alpha}^{R}(t, \tau)\left[n_{\alpha}(\tau)-n_{\alpha}(0)\right],
\end{aligned}
$$

where the average electron occupation number is obtained from

$$
n_{\alpha}(t)=\operatorname{Im}\left[G_{\alpha \alpha}^{<}(t, t)\right],
$$

which is related to that in the original model as $n_{\alpha \uparrow}(t)+$ $n_{\alpha \downarrow}(t)=2 n_{\alpha}(t)$. Notice that in these notations $n_{\alpha}=1$ means the double occupation of sublattice $\alpha$ (electron pairs). A substitution $2 n_{\alpha} \rightarrow n_{\alpha}$ recovers the form of Eq. (12) used in Ref. [37], where $n_{\alpha}=1$ corresponds to the half-filling.

The occupation numbers as well as the phonon displacement are used to trace the dynamics of the metal-insulator transition. The insulating phase with the CDW is characterized by a generally nonsymmetric occupation $n_{0} \neq n_{1}$ as well as by the stationary lattice distortion manifested in the nonzero phonon displacement averages, $\bar{X}_{0}=-\bar{X}_{1} \neq 0$. The metallic phase does not have the lattice distortion and has no difference between the charge distribution, $\bar{X}_{0}=\bar{X}_{1}=0$ and $n_{0}=n_{1}=0.5$.

We also analyze the dynamics of the electron spectral function

$$
N(\omega, t)=-\frac{1}{\pi} \operatorname{Im}\left[\int_{0}^{t_{\infty}} d \tau e^{i \omega \tau} \sum_{\alpha} G_{\alpha \alpha}^{R}(t+\tau, t)\right],
$$

which give us the single-particle DOS and the gap (the choice of $t_{\infty}$ is dictated by the numerical efficiency).

Equations (4) are solved numerically using solvers implemented in the Non-Equilibrium Systems Simulation Library (NESSI) [37] which were successfully used to study the dynamics of various correlated lattice systems, in particular, excited Mott insulators [38,39] and periodically driven cold 
atom systems [24]. In this work, we extended resources of this library to the case of the two site impurities and the matrix Dyson equations.

One notes that the initial Hamiltonian (2) is degenerate with respect to the electron sublattice occupation. In order to obtain the temperature equilibrium CDW state with the broken symmetry, we adopt the method of quasiaverages [40]. Following this method, the symmetry is lowered initially by introducing a small difference between the sublattice chemical potentials. During a subsequent time evolution this symmetry breaking contribution is removed, however, the system evolves towards the equilibrium CDW state. The latter is used as the initial state to obtain the time evolution for the driven system.

\section{Material parameters}

As noted above the hopping matrix elements of the TB model are extracted from the $a b$ initio calculations. The effective matrix elements of the Bethe lattice are obtained using two conditions: (1) the width of the band is equal to that of the TB model $W \simeq 6 \mathrm{eV}$, and (2) the shape of the DOS for the Bethe lattice model should be as close as possible to the TB model. This yields $t_{\|}=1.0 \mathrm{eV}$ and $t_{\perp}=0.5 \mathrm{eV}$ for the intra- and interlattice hopping matrix elements.

Further, the phonon mode frequency is set to $\omega_{\mathrm{ph}}=$ 70me, which corresponds to the Raman breathing mode of $\mathrm{BaBiO}_{3}[41,42]$. The strength of the electron-phonon coupling is estimated from the value of the gap in the single-particle DOS $\Delta \simeq 2.5 \mathrm{eV}$. This yields the coupling constant $g=$ $0.35 \mathrm{eV}$ which is close to $g=0.43 \mathrm{eV}$, reported earlier [27]. In the calculations we consider the case of zero doping with a half-filled band. The calculations are done at room temperature $T=290 \mathrm{~K}$.

In what follows, we investigate the dependence of the dynamics of the system on the frequency $\omega_{0}$ and intensity of the excitation laser pulse. We consider a broad interval of optical frequencies $1 \mathrm{eV} \lesssim \hbar \omega_{0} \lesssim 4 \mathrm{eV}$. For the excitation intensity, we use the dimensionless parameter $A_{0}=e a|E| /\left(\omega_{0} c \hbar\right)$, related to the field amplitude $|E|$, where $a=4.34 \AA$ is the lattice constant of the BBO structure, $e$ is the elementary charge, $c$ is the speed of light. We consider relatively high intensities with the field amplitude $|E| \sim \Delta / e a$. This corresponds to the laser intensity $I_{0}=|E|^{2} / Z_{0} \simeq 10^{13} \mathrm{~W} / \mathrm{cm}^{2}$, where $Z_{0}=376.73 \Omega$ is the vacuum impedance.

\section{DYNAMICS OF A DRIVEN SYSTEM}

The dynamics of the system is investigated for the continuous driving, switched on at $t=0$. The time evolution is obtained by solving the DMFT equations (4) briefly described above. Before the pulse arrival, at $t<0$, the system is in the equilibrium state, calculated self-consistently using the same formalism.

The calculations are done by choosing the frequency $\omega_{0}$ and the amplitude $A_{0}$ of the driving pulse in the ranges shown in Fig. 5. From the complete time evolution, we extract quantities that characterize the transition from the insulating to the metallic state of the system: electronic occupation numbers $n_{\alpha}$ of the sublattice $\alpha$, which yield the electronic (pair)

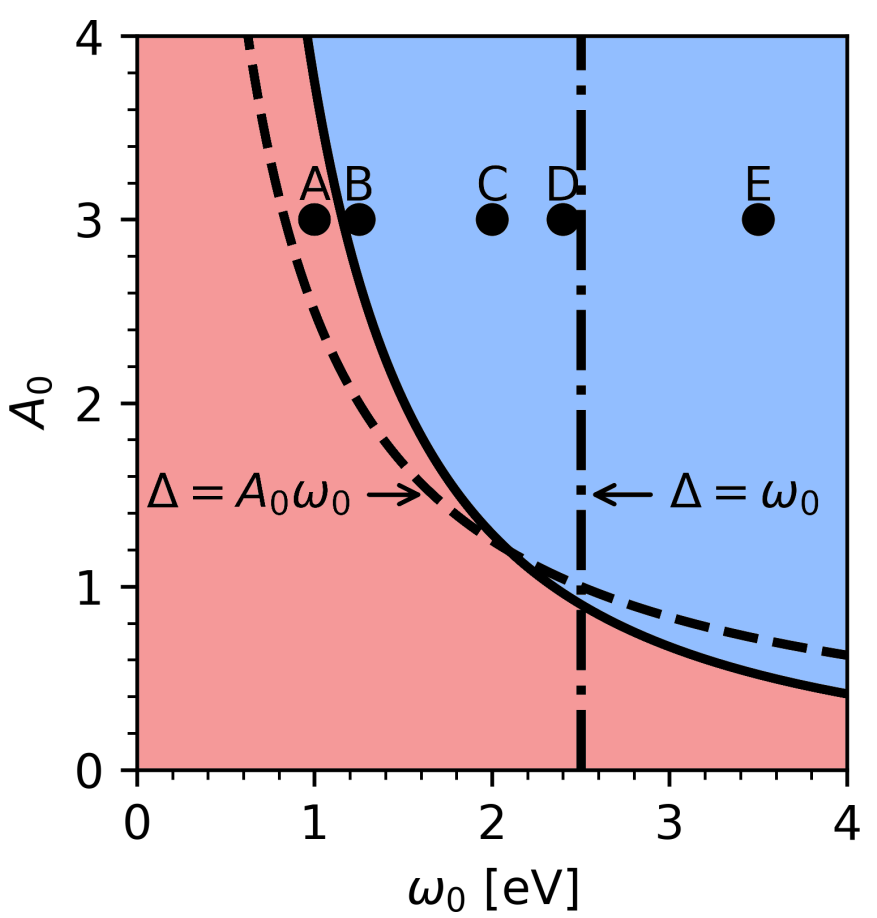

FIG. 5. Phase diagram of types of the time evolution. Red color marks the weak excitation domain, blue color denotes the domain of strong excitation with the transient metal-insulator transition. The dashed line is given by $A_{0} \omega_{0}=\Delta(=2.5 \mathrm{eV})$ is an estimated threshold separating these regimes. The dashed-dotted line is given by $\hbar \omega_{0}=\Delta$. Dynamics, calculated in points A-E is shown in Fig. 6 .

occupation at different octahedra $\mathrm{BiO}_{6}$, the gap $\Delta(t)$ in the single-particle DOS, and the phonon displacement $X_{\alpha}(t)$ (octahedra distortion).

Before the arrival of the pulse the system is in the CDW state where the electronic pairs are shifted to one of the sublattice so that $n_{0}=1$ and $n_{1}=0$, the gap in the DOS has a value $\Delta(0)=2.5 \mathrm{eV}$ and the octahedra are displaced with the amplitudes $X_{0}=0.42 \AA$ and $X_{1}=-0.42 \AA$.

\section{A. General remarks}

Before presenting numerical results it is instructive to discuss general factors that determine the dynamical response of the system.

When the excitation pulse is weak the dynamics is defined mostly by the linear response. The system has a gap in the single-particle DOS, equal to the bipolaron binding energy, and thus the response strongly depends on the relation between the excitation frequency and the gap. Here singleparticle excitations take place when $\hbar \omega_{0}>\Delta$. Analogously to semiconductors the pulse creates electron-hole pairs, which in principle destroys the insulating state making the system conductive. However, the rate of such processes is small so that excited electron-hole pairs recombine back to the ground state and the system remains in the original CDW state with the gap.

The situation becomes more complicated when the intensity of the laser pulse increases and multiphoton assisted tunneling processes start to dominate the time evolution and 
the dynamics becomes nonlinear. When the driving is very strong one can reach a situation where the electronic structure of the system changes so strongly that the lattice distortion disappears together with the gap resulting in the transient metal-insulator transition. One can roughly estimate the onset of such processes as when the field reaches such strength that it overweights the gap-related potential barrier between the different octahedra. Then electrons start moving freely between the sublattices thereby eliminating their asymmetric distribution, lattice static distortion and the spectral gap. This threshold field amplitude is estimated as $e E a \simeq \Delta$, which yields the field strength interval chosen in this work.

The threshold of the driving amplitude given by $A_{0} \omega_{0} \simeq \Delta$ as well as the resonance line $\Delta=\hbar \omega_{0}$ are shown by the dash-dotted and dashed line in the phase diagram of Fig. 5 defining the domains of the dynamics types. In this work, we are interested in the regime of stronger driving where the CDW state of the system can be altered.

\section{B. Dynamics of the electronic subsystem}

Results of the numerical calculations reveal several types of dynamics patterns. Depending of the final state of the system these are divided into two large classes, which occur in the colored domains in Fig. 5. In the regime we call "weak driving" (red domain) the evolution is slow an the system keeps its original insulating state even at very large times. In the regime of "strong driving" (blue domain) the system state rapidly changes to the gapless metallic state. These types interchange rather abruptly when passing the vicinity of the solid black crossover line in Fig. 5. This line represents a summary of the numerical results, however, it is surprisingly close to the simple estimate above, shown by the dashed line in Fig. 5.

Figure 6 shows the time evolution calculated for several representative points $\mathrm{A}-\mathrm{E}$, shown in the diagram in Fig. 5. These points are selected to illustrate typical dynamical patterns in both the weak and the strong driving regimes. We have done the calculations also for many other points in this diagram and they demonstrated very similar results. An interested reader can find the time evolution of the system for additional points on the diagram in Ref. [43].

Panels (A1)-(E1) in Fig. 6 plot the time evolution of the occupation $n_{\alpha}$ for sublattices $\alpha=0,1$. Panels (A2)-(E2) show the spectrum of the dynamics, calculated as the frequency dependent absolute value of the Fourier component of the interlattice current flow $I(\omega) \sim|\boldsymbol{j}(\omega)|^{2}$, where $j(\omega)$ is the Fourier transform of the current $j(t)=\dot{n}_{1}(t)-\dot{n}_{0}(t)$. Finally, panels (A3)-(E3) plot the time evolution of the gap in the single particle spectrum.

At point A (Fig. 5), the system is in the weak driving regime manifested by the weak time dependence of the electron occupation in panel (A1). The corresponding changes in gap, shown in panel (A3), are also small, $\Delta(t)$ saturates when the time increases and shows no sign of abating. At the same time, the spectra of the time evolution in panel (A2) reveals that the dynamics comprises a large nonlinear component with the noticeable contribution of the high harmonics even to the order $n=10$. Appearance of high harmonics in this system is similar to that reported earlier for a strongly driven
Mott insulator [44]. We note in passing that the name "weak driving" for this regime means only that the insulating state does not change in the evolution.

Sharp changes of the dynamical pattern when the system crosses into the strongly driving regime (blue color domain in Fig. 5) are illustrated by the results obtained at point B. Although $\mathrm{B}$ is close to $\mathrm{A}$ the electronic occupation now rapidly decays on the timescale of $50 \mathrm{fs}$ [panel (B1)]. Furthermore, the occupation rapidly approaches a new quasiequilibrium state that has equal electronic occupations, $n_{0}=n_{1}=0.5$, indicating that the CDW is destroyed. This is also seen in panel (B3) which demonstrates that the gap disappears completely after $t_{0} \simeq 40 \mathrm{fs}$. It is worth noting that the gap disappears with time abruptly. Its time evolution follows not a gradual decay but approaches zero as a square root dependence $\left(1-t / t_{0}\right)^{1 / 2}$, reminiscent of a time dependent phase transition.

The spectrum of the occupation dynamics in panel (B2) demonstrates a large contribution of high harmonics. It is worth noting, however, that the relative weight of the higher harmonics decreases in comparison with point A [panel (A2)]. Deeper in the strong driving regime (point $\mathrm{C}$ in Fig. 5) the dynamics remains qualitatively similar to that at point $\mathrm{B}$. Nevertheless, the electronic occupation relaxes faster to its new quasiequilibrium state, the characteristic time for the gap disappearance decreases to $t_{0} \simeq 15 \mathrm{fs}$. One notices a much longer oscillations in the time evolution of the occupation in panel $(\mathrm{C} 1)$. These are related to phonons, which is discussed below. It is important to note that point B is still substantially below the resonance line $\hbar \omega_{0} \ll \Delta$ and, therefore, the dynamics, at least initially till the gap disappearing, is mainly defined by the tunneling through the gap related potential barrier.

The point $\mathrm{D}$ is deeper in the strong driving domain, approaching the resonance condition $\hbar \omega_{0}=\Delta$. Strictly speaking, this resonance corresponds to the single-photon picture in the linear response, whereas in our case the dynamics is highly nonlinear and involve many-photon transitions. Nevertheless, our calculations demonstrate noticeable changes in the dynamics in the vicinity of this line, most likely because of the much increased contributions of the single-particle excitation processes in the initial stage of the time evolution.

The proximity to the resonance $\hbar \omega_{0} \simeq \Delta$ is manifested in a sharp decrease of the decay time in the electron occupation dynamics [panel (D1)], accompanied by a similarly fast disappearance of the gap, $t_{0} \simeq 10 \mathrm{fs}$ [panel (D3)]. Remarkably, the gap reappears after $t_{1} \simeq 30 \mathrm{fs}$. We stress that such reappearance does not take place at point $\mathrm{C}$ even though it is relatively close to $\mathrm{D}$, the longer time calculations demonstrate absence of the gap in this point.

Qualitative changes in the dynamics at point D, in contrast to $\mathrm{C}$, are also manifested in that its spectrum, shown in panel (D2), becomes essentially continuous (although the peaks at the integer harmonics are still visible). The spectrum reveals that the dynamics now has a significant contribution of the slow time evolution with $\omega \sim 0$, which is related to the dynamics of phonons and of the gap reappearance.

With a further increase in the frequency direct excitations over the gap become more and more significant. At point $\mathrm{E}$, the frequency is so high that the laser field moves both electrons at the same time leading to the "overshoot" in the densities, $n_{0}(t)<n_{1}(t)$ [panel (E1)]. The effect of the gap 
(A1)

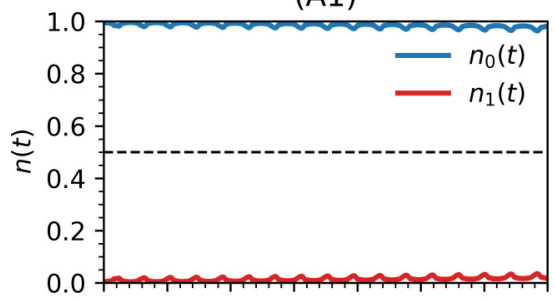

(B1)

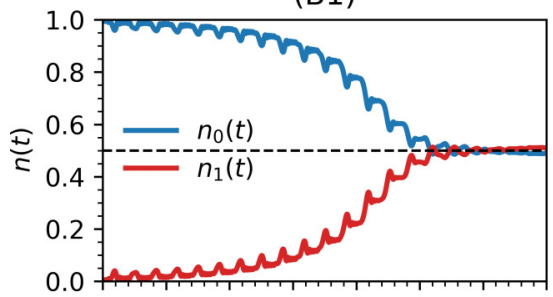

(C1)

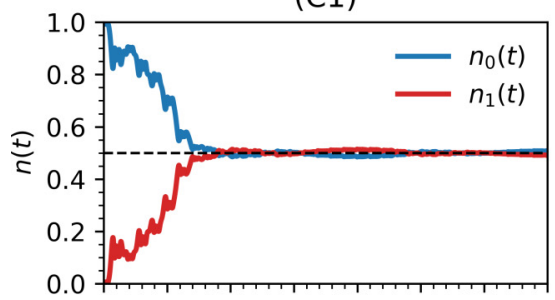

(D1)

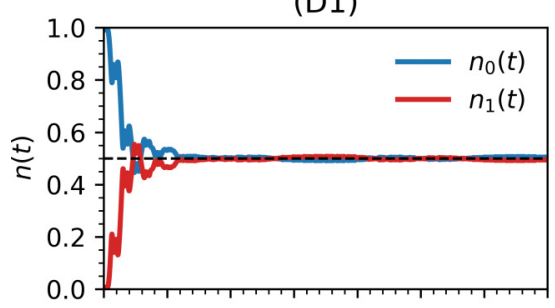

(E1)

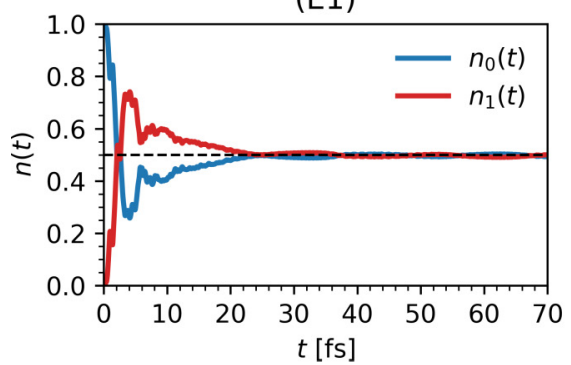

(A2)

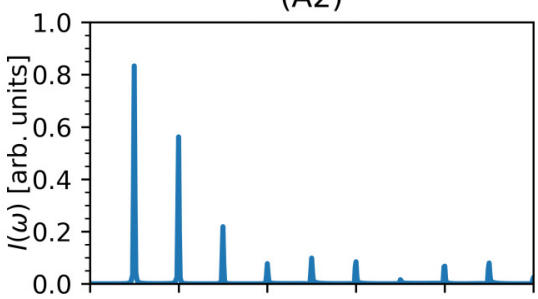

(B2)

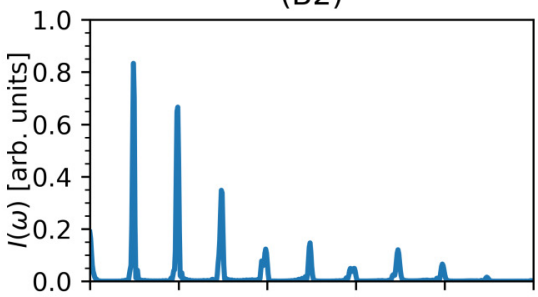

(C2)

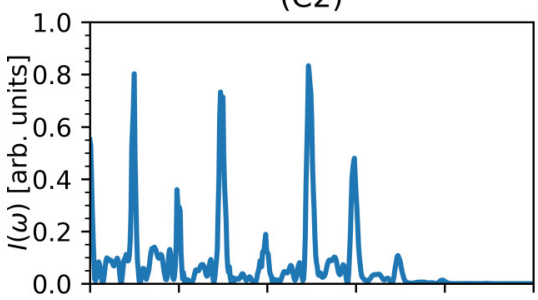

(D2)

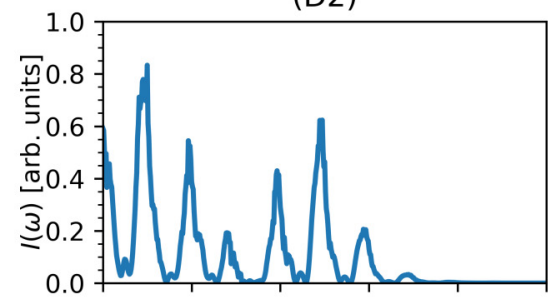

(E2)

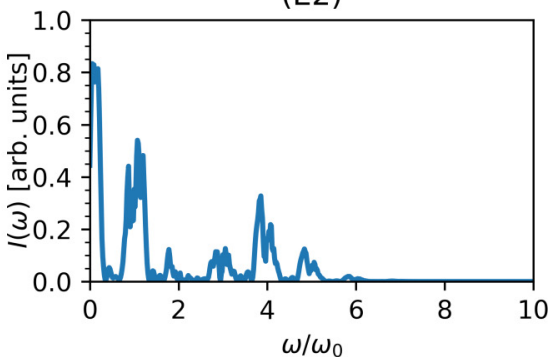

(A3)

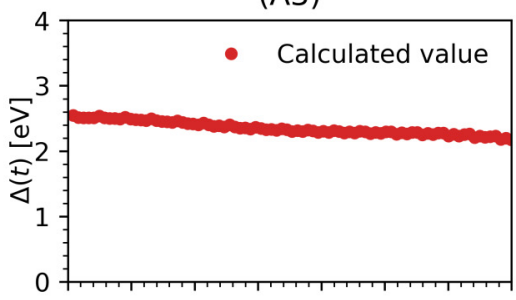

(B3)

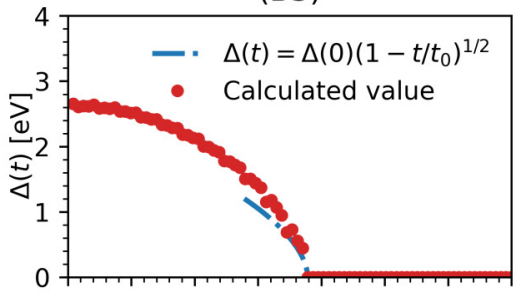

(C3)

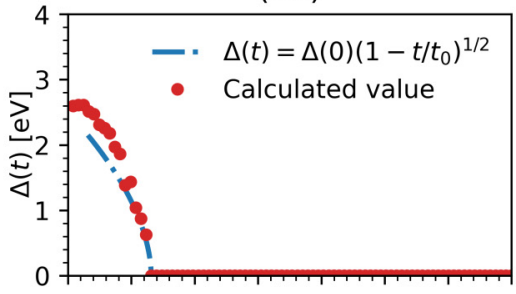

(D3)

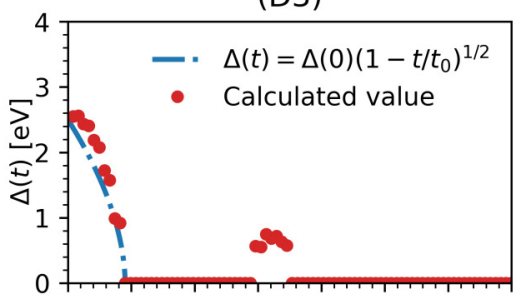

(E3)

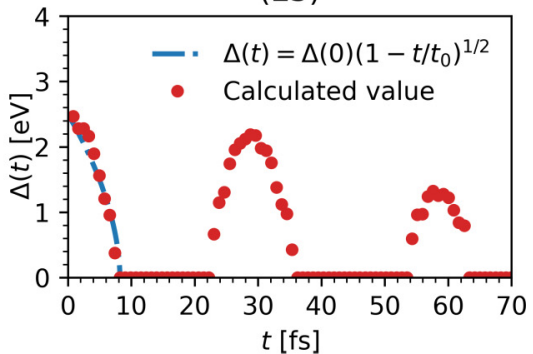

FIG. 6. Time evolution of the driven system calculated for parameters at points A-E in the diagram in Fig. 5 is shown, correspondingly, in rows of panels (A)-(E). Panels (A1)-(E1) plot the time evolution of the electronic occupation in sublattices $\alpha=0,1$. The second panel column A2-E2 shows the spectra $I(\omega)$ of the electron dynamics as follows from the analysis of the Fourier component of the interlattice current flow. Panels (A3)-(E3) plot the time evolution of the dielectric gap. The square root function $\Delta(t)=\Delta(0)\left(1-t / t_{0}\right)^{1 / 2}$ with $\Delta(0)=2.5 \mathrm{eV}$ is used to fit the time dependence of the gap close to its vanishing point.

reappearance becomes more pronounced [panel (E3)], the gap reappears several times before vanishing completely. In contrast the long time calculations at point $\mathrm{D}$ reveal only a single gap reappearance. The spectrum for point $\mathrm{E}$, shown in panel (E2), becomes "more continuous": the integer harmonics peaks are less pronounced. The higher frequency contribution to the spectrum is suppressed comparing to points A-D.
We note that the same results are obtained when the interchange between the weak and strong driving domain goes along other lines in the phase diagram in Fig. 5. The crossover line actually is obtained as a summary of the crossing points at which the dynamic changes. As an example Ref. [43] demonstrates changes in the time evolution along the lines $A_{0}=2$ and $A_{0}=1$, as well as along the vertical line $\omega_{0}=3.5 \mathrm{eV}$. 
(A)

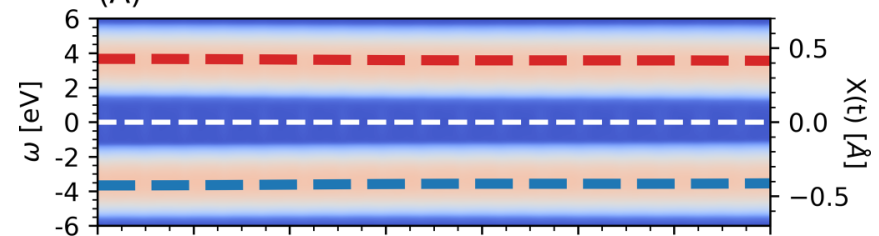

(B)

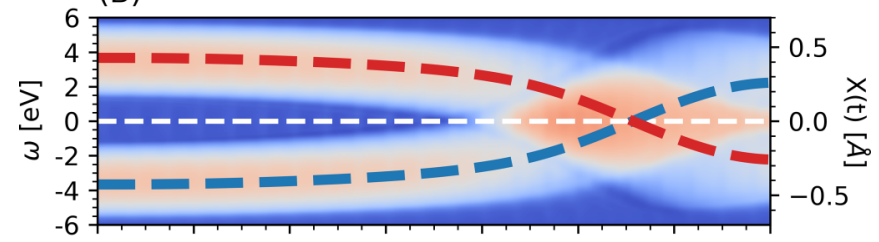

(C)

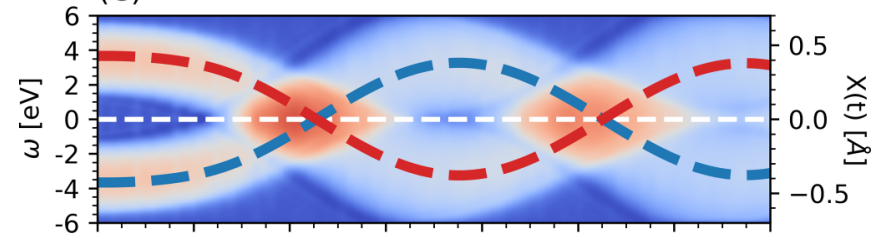

(D)

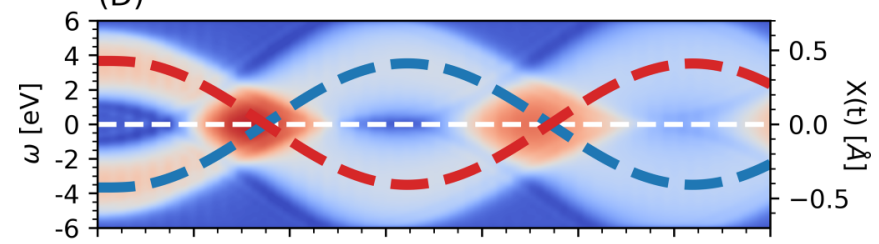

(E)
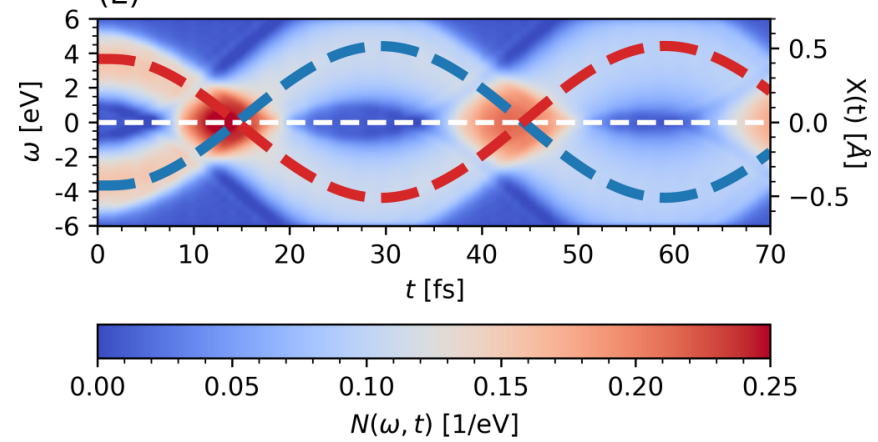

FIG. 7. Time evolution of the electron DOS, shown as the color density plot, and the phonon-induced deformation of the sublattices, shown by two dashed lines. The blue dashed line shows $X_{0}(t)$, and the red $X_{1}(t)$. Panels (A)-(E) correspond to points A-E in Fig. 5.

\section{Spectral gap and deformation dynamics}

A further insight into the system dynamics is obtained by comparing the time evolution of the electronic degrees of freedom with that of the lattice deformation. The color density plot in Fig. 7 shows the time dependence of the electronic DOS superimposed with the deformation amplitude $X_{\alpha}(t)$ mediated by the phonon oscillations. As expected for the CDW state, the dielectric gap in the DOS closely follows the lattice distortion, disappearing precisely at points where it vanishes, $X_{\alpha}=0$.

The gap reappearance decays on the timescale of $\sim 100 \mathrm{fs}$, whereas the phonon oscillations do not decay because the driving constantly pumps energy into the system. The phonon decay sets in when the driving is switched off. It has to be

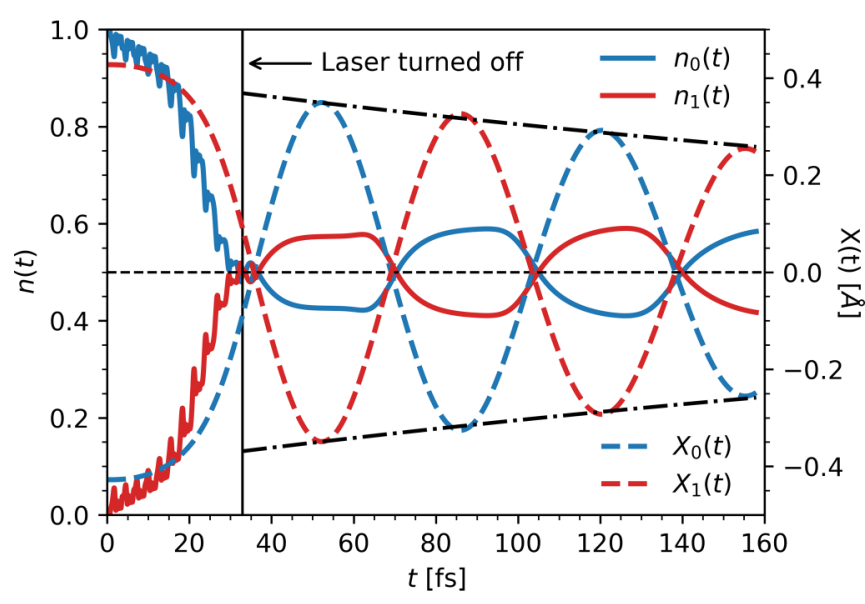

FIG. 8. The dynamical response of the system at point $\mathrm{C}$ in Fig. 5, the laser field is turned off at the phase transition $t_{0}=33 \mathrm{fs}$. Time evolution of the occupation of sites " 0 " and " 1 " is shown by solid lines, lattice displacements are represented by the dashed lines, the dash-dotted line gives the decaying envelope of the phonon displacement.

noted that the Holstein model does not account for mechanisms for the phonon relaxation, and thus phonons decay only via their coupling to electrons. It should also be noted that in this work we are in the limit of very strong phonon-electron coupling. In this case, we are in the limit of the reduced phonon relaxations in comparison to that for electrons, as were reported earlier for the Holstein model [35].

This is shown in Fig. 8, where the laser destroys the CDW state and then is turned off at $t=33 \mathrm{fs}$. The phonon dynamics then induces oscillations in the electronic occupations, which decay gradually with the characteristic time of $\tau_{\mathrm{ph}}=350 \mathrm{fs}$. However, the new equilibrium state is not the original insulating CDW but is the metallic state with $n_{0}=n_{1}=0.5$ without the lattice distortion.

\section{CONCLUSIONS}

This work studied ultrafast dynamics of undoped $\mathrm{BaBiO}_{3}$, excited by a strong continuous laser pulse, with a goal to investigate the laser-induced transition between insulating and metallic phases. The material is described by the Holstein model. The dynamics is obtained using the approach that combines the DMFT and the self-consistent Migdal approximation for the self-energy. The method takes into account quantum coherences and allows to calculate time evolution on the ultrafast timescales. To simplify the calculations the tight-binding model for the electronic states is mapped on the double Bethe lattice model, for which the DMFT equations are greatly simplified.

The dynamics was calculated in a wide range of optical frequencies and excitation intensities. The calculations demonstrated two qualitatively different regimes: the weak driving regime, where the insulating phase remains intact, and the strong driving one, in which the system undergoes a transient change into the metallic state without CDW and lattice deformations. The transition between the two regimes 
is reliably estimated from the condition the driving field overcomes the gap in the single particle DOS.

The insulating state disappears abruptly: the gap vanishes with time by following the square root law, typical for phase transitions. The transition is of purely quantum nature, it is caused not by the temperature, but by the coherent driven dynamics of electron and phonon degrees of freedom. It is not connected to the nonlinearity: in both cases, the dynamics can be highly nonlinear producing a large number of higher harmonics.

Typical characteristic times of the relaxations in the electronic subsystem and that of the insulator-metal transition are found to be in the range of tens of fs, considerably smaller than the characteristic phonon times. Thus the metallic state is achieved on the background of strongly oscillating lattice. However, those oscillations were found to affect the electronic states in a surprising way, causing a periodical reappearance of the gap. This takes place when the driving frequency exceeds the gap. The reappearance time is locked to the period of the lattice oscillations, although the amplitude of the reappearance decays much faster. We also noted that when the laser frequency exceeds the gap the spectrum of the dynamics becomes continuous, although the peaks related to the higher harmonics remain. In this case the spectrum of the electronic dynamics acquires a significant low frequency component, related to the gap reappearance.

Although the adopted model is simplified it is frequently used in the studies of similar structures and is expected to capture the essential physics of the metal-insulator transition, especially on the ultrafast timescales where other relaxation processes are not important. The model parameters were chosen to match those of the real material. In the calculations we have exploited experimentally available laser frequencies and intensities. We also found that the appearance of different regimes can be understood using simple intuitive estimations, which are, nevertheless, quantitatively not very far from those obtained by the calculations, speaking in favor that the results can describe real dynamics and the transient metal-insulator transition in strongly driven $\mathrm{BaBiO}_{3}$ compounds.

\section{ACKNOWLEDGMENTS}

The work was supported by the Russian Foundation for Basic Research under the Project 18-02-40001 mega. Y.Z. also thanks for support the Deutsche Forschungsgemeinschaft under the Project-ID 314695032-SFB 1277.
[1] A. Sleight, J. Gillson, and P. Bierstedt, High-temperature superconductivity in the $\mathrm{BaPb}_{1-\mathrm{x}} \mathrm{Bi}_{x} \mathrm{O}_{3}$ systems, Solid State Commun. 17, 27 (1975).

[2] L. F. Mattheiss, E. M. Gyorgy, and D. W. Johnson, Superconductivity above $20 \mathrm{~K}$ in the Ba-K-Bi-O system, Phys. Rev. B 37, 3745 (1988)

[3] A. P. Menushenkov, K. V. Klementev, A. V. Kuznetsov, and M. Y. Kagan, Superconductivity in $\mathrm{Ba}_{1-x} \mathrm{~K}_{x} \mathrm{BiO}_{3}$ : Possible scenario of spatially separated Fermi-Bose mixture, J. Exp. Theor. Phys. 93, 615 (2001).

[4] A. Menushenkov, K. Klementev, A. Kuznetsov, and M. Kagan, An interplay between spatially separated Fermi and Bose subsystems and superconductivity in perovskite-like oxides, Phys. B: Condens. Matter 312-313, 31 (2002).

[5] A. P. Menushenkov, A. V. Kuznetsov, K. V. Klementiev, and M. Y. Kagan, Fermi-Bose mixture in $\mathrm{Ba}(\mathrm{K}) \mathrm{BiO}_{3}$ superconducting oxide, J. Supercond. Novel Magn. 29, 701 (2016).

[6] A. P. Menushenkov and K. V. Klementev, Extended x-ray absorption fine-structure indication of a double-well potential for oxygen vibration in $\mathrm{Ba}_{1-x} \mathrm{~K}_{x} \mathrm{BiO}_{3}$, J. Phys.: Condens. Matter 12, 3767 (2000).

[7] S. Uchida, K. Kitazawa, and S. Tanaka, Superconductivity and metal-semiconductor transition in $\mathrm{BaPb}_{1-x} \mathrm{Bi}_{x} \mathrm{O}_{3}$, Phase Transitions 8, 95 (1987).

[8] A. W. Sleight, Bismuthates: $\mathrm{BaBiO}_{3}$ and related superconducting phases, Physica C 514, 152 (2015).

[9] N. C. Plumb, D. J. Gawryluk, Y. Wang, Z. Ristić, J. Park, B. Q. Lv, Z. Wang, C. E. Matt, N. Xu, T. Shang, K. Conder, J. Mesot, S. Johnston, M. Shi, and M. Radović, Momentum-Resolved Electronic Structure of the High- $T_{c}$ Superconductor Parent Compound $\mathrm{BaBiO}_{3}$, Phys. Rev. Lett. 117, 037002 (2016).

[10] C. M. Varma, Missing Valence States, Diamagnetic Insulators, and Superconductors, Phys. Rev. Lett. 61, 2713 (1988).
[11] I. Hase and T. Yanagisawa, Madelung energy of the valenceskipping compound $\mathrm{BaBiO}_{3}$, Phys. Rev. B 76, 174103 (2007).

[12] A. P. Menushenkov, K. V. Klement'ev, P. V. Konarev, and A. A. Meshkov, Anharmonicity and superconductivity in $\mathrm{Ba}_{0.6} \mathrm{~K}_{0.4} \mathrm{BiO}_{3}$, J. Exp. Theor. Phys. Lett. 67, 1034 (1998).

[13] J. F. Federici, B. I. Greene, E. H. Hartford, and E. S. Hellman, Optical characterization of excited states in $\mathrm{BaBiO}_{3}$, Phys. Rev. B 42, 923 (1990).

[14] S. Tajima, S. Uchida, A. Masaki, H. Takagi, K. Kitazawa, S. Tanaka, and A. Katsui, Optical study of the metalsemiconductor transition in $\mathrm{BaPb}_{1-x} \mathrm{Bi}_{x} \mathrm{O}_{3}$, Phys. Rev. B 32 6302 (1985).

[15] H. Sato, S. Tajima, H. Takagi, and S. Uchida, Optical study of the metal-insulator transition on $\mathrm{Ba}_{1-x} \mathrm{~K}_{x} \mathrm{BiO}_{3}$ thin films, Nature (London) 338, 241 (1989).

[16] J. Tang, Z. Zou, and J. Ye, Efficient photocatalysis on $\mathrm{BaBiO}_{3}$ driven by visible light, J. Phys. Chem. C 111, 12779 (2007).

[17] D. Nicoletti, E. Casandruc, D. Fu, P. Giraldo-Gallo, I. R. Fisher, and A. Cavalleri, Anomalous relaxation kinetics and chargedensity-wave correlations in underdoped $\mathrm{BaPb}_{1-x} \mathrm{Bi}_{x} \mathrm{O}_{3}$, Proc. Natl. Acad. Sci. USA 114, 9020 (2017).

[18] M. Buzzi, M. Först, R. Mankowsky, and A. Cavalleri, Probing dynamics in quantum materials with femtosecond x-rays, Nat. Rev. Mater. 3, 299 (2018).

[19] B. Moritz, A. F. Kemper, M. Sentef, T. P. Devereaux, and J. K. Freericks, Electron-Mediated Relaxation Following Ultrafast Pumping of Strongly Correlated Materials: Model Evidence of a Correlation-Tuned Crossover between Thermal and Nonthermal States, Phys. Rev. Lett. 111, 077401 (2013).

[20] A. F. Kemper, M. A. Sentef, B. Moritz, J. K. Freericks, and T. P. Devereaux, Effect of dynamical spectral weight redistribution on effective interactions in time-resolved spectroscopy, Phys. Rev. B 90, 075126 (2014). 
[21] M. Sentef, A. F. Kemper, B. Moritz, J. K. Freericks, Z.-X. Shen, and T. P. Devereaux, Examining Electron-Boson Coupling using Time-Resolved Spectroscopy, Phys. Rev. X 3, 041033 (2013).

[22] D. Golež, L. Boehnke, M. Eckstein, and P. Werner, Dynamics of photodoped charge transfer insulators, Phys. Rev. B 100, 041111(R) (2019).

[23] S. Mor, M. Herzog, D. Golež, P. Werner, M. Eckstein, N. Katayama, M. Nohara, H. Takagi, T. Mizokawa, C. Monney, and J. Stähler, Ultrafast Electronic Band Gap Control in an Excitonic Insulator, Phys. Rev. Lett. 119, 086401 (2017).

[24] K. Sandholzer, Y. Murakami, F. Görg, J. Minguzzi, M. Messer, R. Desbuquois, M. Eckstein, P. Werner, and T. Esslinger, Quantum Simulation Meets Nonequilibrium Dynamical Mean-Field Theory: Exploring the Periodically Driven, Strongly Correlated Fermi-Hubbard Model, Phys. Rev. Lett. 123, 193602 (2019).

[25] A. Georges, G. Kotliar, W. Krauth, and M. J. Rozenberg, Dynamical mean-field theory of strongly correlated fermion systems and the limit of infinite dimensions, Rev. Mod. Phys. 68, 13 (1996).

[26] G. Kotliar, S. Y. Savrasov, K. Haule, V. S. Oudovenko, O. Parcollet, and C. A. Marianetti, Electronic structure calculations with dynamical mean-field theory, Rev. Mod. Phys. 78, 865 (2006).

[27] A. Khazraie, K. Foyevtsova, I. Elfimov, and G. A. Sawatzky, Oxygen holes and hybridization in the bismuthates, Phys. Rev. B 97, 075103 (2018).

[28] S. Tajima, S. Uchida, A. Masaki, H. Takagi, K. Kitazawa, S. Tanaka, and $\mathrm{S}$. Sugai, Electronic states of $\mathrm{BaPb}_{1-x} \mathrm{Bi}_{x} \mathrm{O}_{3}$ in the semiconducting phase investigated by optical measurements, Phys. Rev. B 35, 696 (1987).

[29] D. T. Harris, N. G. Campbell, C. Di, J.-M. Park, L. Luo, H. Zhou, G.-Y. Kim, K. Song, S.-Y. Choi, J. Wang, M. S. Rzchowski, and C. B. Eom, Charge density wave modulation in superconducting $\mathrm{BaPbO}_{3} / \mathrm{BaBiO}_{3}$ superlattices, Phys. Rev. B 101, 064509 (2020).

[30] S. Ciuchi and F. de Pasquale, Charge-ordered state from weak to strong coupling, Phys. Rev. B 59, 5431 (1999).

[31] G. Moeller, V. Dobrosavljević, and A. E. Ruckenstein, RKKY interactions and the Mott transition, Phys. Rev. B 59, 6846 (1999).
[32] H. Hafermann, M. I. Katsnelson, and A. I. Lichtenstein, Metal-insulator transition by suppression of spin fluctuations, Europhys. Lett. 85, 37006 (2009).

[33] M. Harland, S. Brener, M. I. Katsnelson, and A. I. Lichtenstein, Exactly solvable model of strongly correlated d-wave superconductivity, Phys. Rev. B 101, 045119 (2020).

[34] M. Harland, M. I. Katsnelson, and A. I. Lichtenstein, Plaquette valence bond theory of high-temperature superconductivity, Phys. Rev. B 94, 125133 (2016).

[35] Y. Murakami, P. Werner, N. Tsuji, and H. Aoki, Interaction quench in the Holstein model: Thermalization crossover from electron- to phonon-dominated relaxation, Phys. Rev. B 91, 045128 (2015).

[36] Y. Murakami, P. Werner, N. Tsuji, and H. Aoki, Multiple amplitude modes in strongly coupled phonon-mediated superconductors, Phys. Rev. B 93, 094509 (2016).

[37] M. Schüler, D. Golež, Y. Murakami, N. Bittner, A. Herrmann, H. U. Strand, P. Werner, and M. Eckstein, NESSi: The non-equilibrium systems simulation package, Comput. Phys. Commun. 257, 107484 (2020).

[38] M. Eckstein and P. Werner, Thermalization of a pump-excited Mott insulator, Phys. Rev. B 84, 035122 (2011).

[39] M. Ligges, I. Avigo, D. Golež, H. U. R. Strand, Y. Beyazit, K. Hanff, F. Diekmann, L. Stojchevska, M. Kalläne, P. Zhou, K. Rossnagel, M. Eckstein, P. Werner, and U. Bovensiepen, Ultrafast Doublon Dynamics in Photoexcited 1T-TaS 2 , Phys. Rev. Lett. 120, 166401 (2018).

[40] N. N. Bogolubov Jr., Quantum Statistical Mechanics (World Scientific, Singapore, 2014).

[41] S. Sugai, S. Uchida, K. Kitazawa, S. Tanaka, and A. Katsui, Lattice Vibrations in the Strong Electron-Phonon-Interaction System $\mathrm{BaPb}_{1-x} \mathrm{Bi}_{x} \mathrm{O}_{3}$ Studied by Raman Scattering, Phys. Rev. Lett. 55, 426 (1985).

[42] S. Tajima, M. Yoshida, N. Koshizuka, H. Sato, and S. Uchida, Raman-scattering study of the metal-insulator transition in $\mathrm{Ba}_{1-x} \mathrm{~K}_{x} \mathrm{BiO}_{3}$, Phys. Rev. B 46, 1232 (1992).

[43] See Supplemental Material at http://link.aps.org/supplemental/ 10.1103/PhysRevResearch.2.043207 for the dynamics at additional points on the diagram.

[44] Y. Murakami, M. Eckstein, and P. Werner, High-Harmonic Generation in Mott Insulators, Phys. Rev. Lett. 121, 057405 (2018). 